\title{
Préparation des fonctions sous-analytiques globales et lieu d'analyticité
}

\author{
par JEAN-MARIE LION (Dijon)
}

\begin{abstract}
We give a new proof of Kurdyka-Tamm's theorem on the analytic locus of a subanalytic function.

0. Introduction. L'objet de ce travail est de donner une nouvelle démonstration du théorème de Tamm-Kurdyka [Ta], [Ku] sur la sous-analyticité du lieu d'analyticité des fonctions sous-analytiques globales (voir aussi $[\mathrm{BM}]$ et $[\mathrm{DM}])$. Nous allons déduire élémentairement ce résultat d'un théorème de préparation des fonctions sous-analytiques globales [Par], [LR]. Le lecteur peut très facilement adapter la méthode exposée ici pour retrouver le résultat de van den Dries et Miller [DM] sur le lieu de régularité des $x^{\lambda}$-fonctions. L'ingrédient à ajouter est le théorème de préparation pour les $x^{\lambda}$-fonctions exposé dans [LR].
\end{abstract}

I. Définitions et résultats. Un sous-ensemble $X$ de $\mathbb{R}^{d}$ est un sousensemble semi-analytique si pour tout point $a$ de $\mathbb{R}^{d}$ il existe un voisinage ouvert $U$, un entier $p$ et des fonctions analytiques $\left(f_{i, j}\right)_{i, j \leq p}$ et $\left(g_{i, j}\right)_{i, j \leq p}$ définies sur $U$ tels que

$$
X \cap U=\bigcup_{i \leq p}\left(\bigcap_{j \leq p}\left\{x \in U \mid f_{i, j}(x)=0, g_{i, j}(x)>0\right\} .\right)
$$

Un sous-ensemble $X$ d'une variété analytique $M$ de dimension $d$ est un semi-analytique dans $M$ si pour tout plongement analytique $\phi$ de $\mathbb{R}^{d}$ dans $M$, l'ensemble $\phi^{-1}(X)$ est un semi-analytique de $\mathbb{R}^{d}$. Nous renvoyons le lecteur aux travaux de Łojasiewicz [Ło1,2] pour les propriétés des semi-analytiques.

On munit la droite projective réelle $\mathbb{P}_{1}$ de sa structure algébrique usuelle qui est donnée par l'équivalence " $[x: 1] \sim[1: y]$ ssi $x y=1$ ”. La droite réelle

1991 Mathematics Subject Classification: Primary 32B20.

Key words and phrases: density, subanalytic subsets, pfaffian subsets. 
$\mathbb{R}$ est algébriquement plongée dans $\mathbb{P}_{1}$. Un sous-ensemble $X$ de $\mathbb{R}^{d}$ est un sous-analytique global s'il existe un entier $m$ et un ensemble $Y$ inclus dans $\mathbb{R}^{d+m}$ qui est semi-analytique dans $\mathbb{P}_{1}^{d+m}$ tel que $X=\pi(Y)$ où $\pi$ désigne la projection canonique de $\mathbb{R}^{d+m}$ sur $\mathbb{R}^{d}$.

Gabrielov [Ga] a démontré que les sous-analytiques globaux possèdent la propriété fondamentale suivante : la différence de deux sous-analytiques globaux est un sous-analytique global.

Une fonction $f$ définie sur un sous-ensemble $U$ de $\mathbb{R}^{d}$ et à valeurs dans $\mathbb{R}$ est sous-analytique globale si son graphe est un sous-analytique global de $\mathbb{R}^{d+1}$. On déduit du théorème du complémentaire que le domaine de définition d'une fonction sous-analytique globale, son lieu de discontinuité, le lieu où elle n'est pas $C^{N}, N \in \mathbb{N}$ étant un entier fixé, sont des sousanalytiques globaux. Le résultat de Tamm et Kurdyka dont on va proposer une démonstration élémentaire généralise cette dernière propriété.

ThÉOrème DE TAMM-Kurdyka [Ta], $[\mathrm{Ku}]$. Soit $f$ une fonction sousanalytique globale définie sur un sous-ensemble $U$ de $\mathbb{R}^{d}$. Le lieu $S_{\omega}$ des points où $f$ n'est pas analytique est un sous-analytique global. Il existe un entier $N$ tel que $S_{\omega}$ soit égal au lieu $S_{N}$ des points où $f$ n'est pas de classe $C^{N}$.

Le théorème de préparation des fonctions sous-analytiques globales de [Par] et de [LR] va nous permettre de prouver cet énoncé. Dans le chapitre suivant nous en donnons une version adaptée au problème.

II. Quelques propriétés des objets sous-analytiques. Enonçons deux conséquences immédiates du théorème du complémentaire.

- Soient $X_{0}, \ldots, X_{r}$ des sous-analytiques globaux de $\mathbb{R}^{n+1}$. Il existe une partition finie $\mathcal{P}$ de $\mathbb{R}^{n+1}$ en cylindres sous-analytiques globaux adaptée à la famille $X_{0}, \ldots, X_{r}$ : si $C \in \mathcal{P}$ et si $i=0, \ldots, r$ alors $C \subset X_{i}$ ou $C \cap X_{i}=\emptyset$ et $C$ est de la forme $\{y \in B, \phi(y)<z<\psi(z)\}$ ou de la forme $\{(y, \phi(y)) \mid y \in B\}$ où $B$ est un sous-analytique global de $\mathbb{R}^{n}$ et $\phi<\psi$ sont des fonctions sous-analytiques (éventuellement $\phi=-\infty$ ou $\psi=+\infty$ ).

- Soient $\phi_{0}, \ldots, \phi_{r}$ des fonctions sous-analytiques globales définies sur un sous-analytique global $U$ de $\mathbb{R}^{n}$. Quitte à partitionner finiment $U$ en sousanalytiques globaux et à réindexer la famille, on peut la supposer ordonnée.

Les fonctions sous-analytiques admettent la présentation suivante.

Proposition 1 (d'après [Par] et [LR]). Soient $\phi_{0}, \ldots, \phi_{r}$ des fonctions sous-analytiques globales bornées définies sur un sous-analytique global $Y \times] 0,1[$ inclus dans $] 0,1\left[^{n+1}\right.$. Il existe une partition finie $\mathcal{S}$ en sous-analytiques globaux de $Y$ telle que pour chaque sous-analytique global $B$ de $\mathcal{S}$ l'énoncé suivant est vérifié : il existe des entiers $k \in \mathbb{N}, q \in \mathbb{N}$, et $p_{0}, \ldots, p_{r} \in$ 
$\mathbb{N}$, des fonctions sous-analytiques globales $a, \psi_{0}, \ldots, \psi_{k}, A_{0}, \ldots, A_{r}$ définies sur $B$, à valeurs dans ]0, 1 [ et avec a $<\psi_{0}$, des réels $M_{0}, \ldots, M_{r}$, des séries entières $U_{0}, \ldots, U_{r}$ définies au voisinage du polydisque $\Delta_{k+1}=\left\{\left|t_{0}\right|, \ldots,\left|t_{k}\right|\right.$ $\leq 1\}$ de $\mathbb{C}^{k+1}$ et à valeurs dans $[1 / 2,1]$ tels que

$$
\phi_{i}(y, z)=M_{i} \cdot\left(z / \psi_{0}\right)^{p_{i} / q} \cdot A_{i} \cdot U_{i}\left(\left(z / \psi_{0}\right)^{1 / q}, \psi_{1}, \ldots, \psi_{k}\right)
$$

si $i=0, \ldots, r$ et $(y, z)$ appartient au cylindre $\{y \in B, z \in] 0, a(y)[\}$.

Cette proposition est voisine de résultats de [Paw] et de [ŁTZ]. Elle permet de comprendre le défaut d'analyticité par rapport à une variable d'une fonction sous-analytique globale. Elle se déduit du théorème 1 de [LR] de la façon suivante.

Préparons simultanément les fonctions $\phi_{0}, \ldots, \phi_{r}$ (théorème 1 de $[\mathrm{LR}]$ et affirmation $1.1 .5 \mathrm{de}[\mathrm{LR}]$ ). On obtient une partition finie $\mathcal{T}$ en cylindres sous-analytiques globaux de $Y \times] 0,1[$. On ne retient que les cylindres de $\mathcal{T}$ de la forme $C=\{(y, z) \mid y \in B, z \in] 0, a(y)[\}$, où $B$ est un sousanalytique global et $a$ une fonction sous-analytique globale définie sur $B$ et strictement positive. Les bases $B$ de ces cylindres forment la partition $\mathcal{S}$ de $Y$. En restriction à chacun de ces cylindres les fonctions $\phi_{i}$ admettent l'écriture voulue : contrairement à la situation générale de [LR], ici $p_{i} \notin \mathbb{Z}_{-}$ et l'unité $U_{i}$ est indépendante d'un terme de la forme $\left(\Psi_{0} / z\right)^{1 / q}$ car sur les cylindres $C$, la variable $z$ est arbitrairement petite et les fonctions $\phi_{i}$ sont bornées.

III. Démonstration du théorème de Tamm-Kurdyka. D'après le théorème du complémentaire, il suffit de traiter le cas d'une fonction sous-analytique globale $f$ définie sur $\mathbb{R}^{d}$, à support dans $] 0,1\left[^{d}\right.$ et bornée.

On note $l_{0}\left(x, x^{\prime}\right)$ la fonction sous-analytique globale définie sur $] 0,1\left[^{d} \times\right.$ ]$-1,1\left[{ }^{d}\right.$ par $l_{0}\left(x, x^{\prime}\right)=f\left(x+x^{\prime}\right)$. Pour démontrer le théorème on va montrer l'existence d'un entier $N$ et de fonctions sous-analytiques globales $l_{1}\left(x, x^{\prime}\right)$, $\ldots, l_{d}\left(x, x^{\prime}\right)$ définies sur $] 0,1\left[^{d} \times\right]-1,1^{d}$ et vérifiant les propriétés suivantes. Soit $i=1, \ldots, d$ et $\left.\left(x, x_{1}^{\prime}, \ldots, x_{d-i}^{\prime}\right) \in\right] 0,1\left[^{d} \times\right]-1,1\left[^{d-i}\right.$ :

- Il existe $\varepsilon>0$ tel que la fonction

$$
\left(x_{d-i+1}^{\prime}, \ldots, x_{d}^{\prime}\right) \mapsto l_{i}\left(x, x_{1}^{\prime}, \ldots, x_{d-i}^{\prime}, x_{d-i+1}^{\prime}, \ldots, x_{d}^{\prime}\right)
$$

est analytique sur le polydisque $]-\varepsilon, \varepsilon\left[{ }^{i}\right.$.

- Si la fonction $\left(x_{d-i+1}^{\prime}, \ldots, x_{d}^{\prime}\right) \mapsto l_{i-1}\left(x, x_{1}^{\prime}, \ldots, x_{d-i}^{\prime}, x_{d-i+1}^{\prime}, \ldots, x_{d}^{\prime}\right)$ est de classe $C^{N}$ en $0 \in \mathbb{R}^{i}$ elle est analytique au voisinage de $0 \in \mathbb{R}^{i}$ et elle coïncide avec $\left(x_{d-i+1}^{\prime}, \ldots, x_{d}^{\prime}\right) \mapsto l_{i}\left(x, x_{1}^{\prime}, \ldots, x_{d-i}^{\prime}, x_{d-i+1}^{\prime}, \ldots, x_{d}^{\prime}\right)$ sur le polydisque $]-\varepsilon, \varepsilon\left[{ }^{i}\right.$.

Supposons avoir prouvé l'existence de l'entier $N$ et des fonctions $l_{1}, \ldots, l_{d}$ et finissons la preuve du théorème. Soit $\left.x_{0} \in\right] 0,1\left[{ }^{d}\right.$. Etant données les 
propriétés des fonctions sous-analytiques globales $l_{i}$, les trois conditions suivantes sont équivalentes :

(i) La fonction $f$ est de classe $C^{N}$ en $x_{0}$.

(ii) La fonction $f$ est analytique au voisinage de $x_{0}$.

(iii) La fonction $f$ coïncide avec la fonction $l_{d}\left(x_{0}, x-x_{0}\right)$ au voisinage de $x_{0}$.

Puisque les conditions (i) et (iii) sont des conditions sous-analytiques globales, le théorème est démontré.

IV. Existence des fonctions $l_{1}, \ldots, l_{d}$ et de l'entier $N$. Pour démontrer l'existence des fonctions $l_{1}, \ldots, l_{d}$ et de l'entier $N$ il suffit d'utiliser $d$ fois les trois lemmes qui vont suivre. On déduit $l_{i}$ de $l_{i-1}$ en appliquant les trois lemmes avec $\theta=l_{i-1}, n=2 d-i, n^{\prime}=i-1, y=\left(x, x_{1}^{\prime}, \ldots, x_{d-i}^{\prime}\right), z=$ $x_{d-i+1}^{\prime}$ et $u=\left(x_{d-i+2}^{\prime}, \ldots, x_{d}^{\prime}\right)$. La fonction $l_{i}$ est la fonction $l$ ainsi obtenue. Cette construction est possible car la fonction $l_{0}$ vérifie les hypothèses $\mathrm{du}$ lemme 1 et si $l_{i-1}$ les vérifie c'est aussi le cas pour $l_{i}$. L'entier $N$ est le plus grand des entiers $Q_{i}$ obtenus dans cette construction.

L'objet des trois lemmes et d'étudier le lieu des points d'analyticité par rapport aux $n^{\prime}+1$ dernières variables d'une fonction sous-analytique globale qui est analytique par rapport aux $n^{\prime}$ dernières variables.

LEMme 1. Soit $\theta$ une fonction sous-analytique globale bornée de $] 0,1\left[{ }^{n} \times\right.$ ] $0,1[\times] 0,1\left[^{n^{\prime}}\right.$. On suppose qu'il existe une partition finie $\mathcal{P}$ en sous-analytiques globaux de $] 0,1\left[{ }^{n}\right.$ telle que sur chaque sous-analytique global $Y$ de $\mathcal{P}$, la fonction $\theta$ admet une écriture de la forme suivante : il existe des fonctions sous-analytiques globales $\alpha$ définie sur $Y$ et $\beta, \phi_{0}, \ldots, \phi_{r}$ définies sur $Z=\{(y, z) \mid y \in Y, z \in] 0, \alpha(y)[\}$, toutes à valeurs dans $] 0,1[$ et $\beta<\phi_{0}$, et une série entière $g$ définie au voisinage du polydisque $\Delta_{n^{\prime}+r}=$ $\left\{\left(t_{1}, \ldots, t_{n^{\prime}+r}\right)|| t_{i} \mid \leq 1\right\}$ tels que

$\theta(y, z, u)=g\left(u_{1} / \phi_{0}, \ldots, u_{n^{\prime}} / \phi_{0}, \phi_{1}, \ldots, \phi_{r}\right) \quad$ si $\left.(y, z) \in Z, u \in\right] 0, \beta(y, z)\left[{ }^{n^{\prime}}\right.$.

Alors chaque $Y \in \mathcal{P}$ admet une partition sous-analytique finie $\mathcal{P}_{Y}$ telle que pour chaque sous-analytique global $B$ de $\mathcal{P}_{Y}$ l'énoncé suivant est vérifié : il existe des entiers $k^{\prime} \in \mathbb{N}, q, p \in \mathbb{N}$ et $\varepsilon \in\{-1,1\}$, des fonctions sousanalytiques globales $a, A, \psi_{0}, \ldots, \psi_{k^{\prime}}$ définies sur $B$ et à valeurs dans $] 0,1[$ avec $a<\psi_{0}$, $\alpha$ et $\beta(y, z)<2 A^{\varepsilon} a^{p / q}$ si $0<z<a$ et une série entière $G$ définie au voisinage du polydisque $\Delta_{n^{\prime}+k^{\prime}+1}=\left\{\left(t_{0}, \ldots, t_{n^{\prime}+k^{\prime}}\right)|| t_{i} \mid \leq 1\right\}$ tel que

$$
\theta(y, z, u)=G\left(\left(z / \psi_{0}\right)^{1 / q}, u_{1} /\left(A^{\varepsilon} z^{p / q}\right), \ldots, u_{n^{\prime}} /\left(A^{\varepsilon} z^{p / q}\right), \psi_{1}, \ldots, \psi_{k^{\prime}}\right)
$$

si $(y, z)$ appartient au cylindre $\{y \in B, z \in] 0, a(y)[\}$ et $u \in] 0, \beta(y, z) / 2\left[{ }^{n^{\prime}}\right.$. 
Preuve. Il suffit d'appliquer la proposition 1 aux fonctions $\phi_{0}, \ldots, \phi_{r}$ : on obtient des fonctions $a, \psi_{0}, \ldots, \psi_{k}, A_{0}, \ldots, A_{r}$ et des entiers $q, p_{0}, \ldots, p_{r}$ et $k$. On pose $p=p_{0}, k^{\prime}=k+r+1$ et $\psi_{k+i+1}=A_{i}$ si $i=0, \ldots, r$. En raffinant la partition obtenue, on obtient les inégalités voulues. En particulier, on obtient des cylindres sous-analytiques $\{y \in B, z \in] 0, a(y)[\}$ sur lesquels $\phi_{0}$ admet l'écriture

$$
\phi_{0}(y, z)=M_{0} \cdot\left(z / \psi_{0}\right)^{p / q} \cdot A_{0} \cdot U_{0}\left(\left(z / \psi_{0}\right)^{1 / q}, \psi_{1}, \ldots, \psi_{k}\right)
$$

avec $M_{0} A_{0} / \psi_{0}^{p / q} \leq 1$ ou $\psi_{0}^{p / q} /\left(M_{0} A_{0}\right) \leq 1$. Dans le premier cas on pose $A=M_{0} A_{0} / \psi_{0}^{p / q}$ et $\varepsilon=1$. Dans le second cas on pose $A=\psi_{0}^{p / q} /\left(M_{0} A_{0}\right)$ et $\varepsilon=-1$. Ceci permet d'écrire $\theta$ sous la forme

$$
\theta=g \circ L \circ\left(\left(z / \psi_{0}\right)^{1 / q}, u_{1} /\left(A^{\varepsilon} z^{p / q}\right), \ldots, u_{n^{\prime}} /\left(A^{\varepsilon} z^{p / q}\right), \psi_{1}, \ldots, \psi_{k^{\prime}}\right)
$$

où les fonctions coordonnées de l'application $L=\left(L_{1}, \ldots, L_{n^{\prime}+r}\right)$ sont des séries entières définies au voisinage du polydisque $\Delta_{n^{\prime}+k^{\prime}+1}$ et les fonctions $a, A, \psi_{0}, \ldots, \psi_{k^{\prime}}$ satisfont les conclusions du lemme. La série $G$ est la composée $g \circ L$.

Les notations sont celles du lemme 1. Soit $Y \in \mathcal{P}$. Quitte à raffiner la partition $\mathcal{P}_{Y}$ on peut supposer que tout sous-analytique global $B$ de $\mathcal{P}_{Y}$ dont l'adhérence rencontre $] 0,1\left[^{n-1} \times\{0\}\right.$ est un cylindre de la forme

$$
\left\{\left(y^{\prime}, y_{n}\right) \mid y^{\prime} \in B^{\prime}, 0<y_{n}<\alpha^{\prime}(y)\right\}
$$

où $B^{\prime}$ est un sous-analytique global inclus dans $] 0,1\left[^{n-1}\right.$ et $\alpha^{\prime}$ est une fonction sous-analytique globale définie sur $B^{\prime}$ et strictement positive. On note $\mathcal{P}_{Y}^{\prime}$ la sous-famille de $\mathcal{P}_{Y}$ formée par ces cylindres. Les bases de ces cylindres forment une partition de $] 0,1\left[{ }^{n-1}\right.$.

Le lemme suivant reprend les notations du lemme 1. C'est un lemme de scission analogue au lemme du paragraphe 1.6 de $[\mathrm{LR}]$.

Lemme 2. Soit $Y \in \mathcal{P}$ et $B$ un cylindre de $\mathcal{P}_{Y}^{\prime}$. Il existe des séries entières $L, R_{1}, \ldots, R_{q-1}$ définies au voisinage de $\Delta_{n^{\prime}+k^{\prime}+1}$ et $W_{1}, \ldots, W_{n^{\prime}}$ définies au voisinage de $\Delta_{2 n^{\prime}+k^{\prime}}$ et des fonctions sous-analytiques globales $l_{B}, r_{1}, \ldots, r_{q-1}$ et $w$ à support le polycylindre

$$
\left\{y \in B,|z| \leq a(y),\left|u_{1}\right|, \ldots,\left|u_{n^{\prime}}\right| \leq A^{\varepsilon} \psi_{0}^{p / q}\right\}
$$

telles que si $y \in B, z \in] 0, a(y)[$ et $u \in] 0, \beta(y, z) / 2\left[{ }^{n^{\prime}}\right.$ alors

$$
\theta(y, z, u)=l_{B}(y, z, u)+\sum_{j=1}^{q-1} r_{j}(y, z, u)+w(y, z, u)
$$


avec

$$
\begin{aligned}
& l_{B}(y, z, u)=L\left(z / \psi_{0}, u_{i} /\left(A^{\varepsilon} \psi_{0}^{p / q}\right), \psi_{1}, \ldots, \psi_{k^{\prime}}\right) \\
& r_{j}(y, z, u)=\left(z / \psi_{0}\right)^{j / q} R_{j}\left(z / \psi_{0}, u_{1} /\left(A^{\varepsilon} \psi_{0}^{p / q}\right),\right. \\
&\left.\ldots, z / \psi_{0}, u_{n^{\prime}} /\left(A^{\varepsilon} \psi_{0}^{p / q}\right), \psi_{1}, \ldots, \psi_{k^{\prime}}\right) \\
& w(y, z, u)=\sum_{i=1}^{n^{\prime}}\left(u_{i}^{1 / p} /\left(A^{\varepsilon / p} z^{1 / q}\right)\right) w_{i}(y, z, u) \\
& w_{i}(y, z, u)=W_{i}\left(u_{1}^{1 / p} /\left(A^{\varepsilon / p} z^{1 / q}\right), \ldots, u_{n^{\prime}}^{1 / p} /\left(A^{\varepsilon / p} z^{1 / q}\right),\right. \\
&\left.u_{1}^{1 / p} /\left(A^{\varepsilon / p} \psi_{0}^{1 / q}\right), \ldots, u_{n^{\prime}}^{1 / p} /\left(A^{\varepsilon / p} \psi_{0}^{1 / q}\right), \psi_{1}, \ldots, \psi_{k^{\prime}}\right) .
\end{aligned}
$$

De plus à y fixé, la restriction de $l_{B} \grave{a}\left\{|z| \leq a(y),\left|u_{1}\right|, \ldots,\left|u_{n^{\prime}}\right| \leq A^{\varepsilon} \psi_{0}^{p / q}\right\}$ est analytique.

Preuve. Soit $m=\left(m_{0}, m_{1}, \ldots, m_{n^{\prime}}\right) \in \mathbb{N}^{n^{\prime}+1}$. Il suffit de regrouper les termes de la série $G$ pour construire les nouvelles séries en remarquant que le produit

$$
\left(z / \psi_{0}\right)^{m_{0} / q} \prod_{i=1}^{n^{\prime}}\left(u_{i} / A^{\varepsilon} z^{p / q}\right)^{m_{i}}
$$

peut s'écrire de plusieurs façons. Si $m_{0} \geq p m_{1}+\ldots+p m_{n^{\prime}}$, on l'écrit sous la forme

$$
\left(z / \psi_{0}\right)^{\left(m_{0}-p \sum_{i \geq 1} m_{i}\right) / q} \prod_{i=1}^{n^{\prime}}\left(u_{i} / A^{\varepsilon} \psi_{0}^{p / q}\right)^{m_{i}} .
$$

Ce produit est non-ramifié par rapport à $z$ si et seulement si $q$ divise $m_{0}-$ $\sum_{i \geq 1} m_{i}$. Ce monôme contribue à l'une des séries $L, R_{1}, \ldots, R_{q-1}$ suivant la congruence modulo $q$ de $m_{0}-\sum_{i \geq 1} m_{i}$. Si $m_{0}<p m_{1}+\ldots+p m_{n^{\prime}}$ on l'écrit sous la forme

$$
\prod_{i=1}^{n^{\prime}}\left(u_{i}^{1 / p} /\left(A^{\varepsilon / p} \psi_{0}^{1 / q}\right)\right)^{m_{i}^{\prime}}\left(u_{i}^{1 / p} /\left(A^{\varepsilon / p} z^{1 / q}\right)\right)^{p m_{i}-m_{i}^{\prime}}
$$

ou les $m_{i}^{\prime} \in \mathbb{N}$ sont des entiers vérifiant $m_{i}^{\prime} \leq p m_{i}$ et $m_{1}^{\prime}+\ldots+m_{n}^{\prime}=m_{0}$. Ce monôme contribue à l'une des séries $W_{i^{\prime}}$ telles que $m_{i^{\prime}}^{\prime}<p m_{i^{\prime}}$.

Soit $y$ un point fixé de $B$. On pose $D_{y}=\{(z, u) \mid z \in] 0, a(y)[, u \in$ ] $0, \beta(x, y) / 2\left[^{n^{\prime}}\right\}$. La restriction de la fonction $l_{B}(y, z, u)$ à $D_{y}$ s'étend en un germe de fonction analytique de $(z, u)$. A contrario, les restrictions des fonctions $r_{j}(y, z, u)$ à $D_{y}$ ne s'étendent en des germes de fonctions analytiques de $(z, u)$ que si elles sont nulles. La restriction de la fonction $w(y, z, u)$ à $D_{y}$ ne s'étend en un germe de fonction continue de $(z, u)$ que si elle est nulle. De plus le germe en 0 de la restriction de la fonction $\theta$ à $D_{y}$ coïncide avec 
la restriction d'un germe de fonction analytique si et seulement si la restriction à $D_{y}$ de la différence $\theta(y, z, u)-l_{B}(y, z, u)$ est nulle. Ceci est vérifié si et seulement si les restrictions à $D_{y}$ des fonctions $r_{j}(y, z, u)$ et $w(y, z, u)$ sont nulles. Or, on déduit de la propriété de noethérianité des fonctions analytiques qu'il existe un entier $q^{\prime}$ tel que les séries $R_{j}\left(t_{0}, \ldots, t_{n^{\prime}+k^{\prime}}\right)$ et $W_{i}\left(t_{1}, \ldots, t_{2 n^{\prime}+k^{\prime}}\right)$ sont indépendantes respectivement des $n^{\prime}+1$ premières variables et des $n^{\prime}$ premières variables aux points $\left(0, \ldots, 0, t_{n^{\prime}+1}, \ldots, t_{n^{\prime}+k^{\prime}}\right)$ et $\left(0, \ldots, 0, t_{2 n^{\prime}+1}, \ldots, t_{2 n^{\prime}+k^{\prime}}\right)$ où les dérivées partielles d'ordre inférieur à $q^{\prime}$ par rapport aux $n^{\prime}+1$ premières variables (respectivement d'ordre inférieur à $q^{\prime}-1$ par rapport aux $2 n^{\prime}$ premières variables) sont nulles. Cette affirmation appliquée à la fonction $\theta$ et aux fonctions $r_{j}$ et $w$ permet de conclure à l'énoncé suivant.

LEMME 3. Il existe une partition de $B$ en deux sous-analytiques globaux $R$ et $S$ vérifiant:

- Si $y \in R$, le germe en 0 de la restriction de la fonction $\theta$ à $D_{y}$ coüncide avec la restriction d'un germe de fonction analytique : la restriction à $D_{y}$ de la différence $\theta(y, z, u)-l_{B}(y, z, u)$ est nulle.

- Si $y \in S$, le germe en 0 de la restriction de la fonction $\theta$ à $D_{y}$ ne peut s'étendre en un germe de fonction de classe $C^{q^{\prime}+q}$.

Preuve. Le sous-analytique global $R$ est le lieu d'annulation des fonctions sous-analytiques globales de la forme $c\left(0, \ldots, 0, \psi_{1}, \ldots, \psi_{k^{\prime}}\right)$ où $c$ est une des dérivées partielles indiquées précédemment.

On note $l$ la fonction sous-analytique globale suivante : $l(x)=l_{B}(x)$ s'il existe $Y \in \mathcal{P}$ et $B \in \mathcal{P}_{Y}^{\prime}$ tel que $x \in B, l(x)=0$ sinon. On note $Q$ le plus grand des entiers $q^{\prime}+q$, lorsque $B \in \mathcal{P}_{Y}^{\prime}$ et $Y \in \mathcal{P}$. L'affirmation suivante est un corollaire du lemme $3:$ Soit $y \in] 0,1{ }^{n}$ le germe en 0 de la fonction $(z, v) \mapsto \theta(y, z, v)$ est la restriction d'un germe de fonction analytique ssi la différence $(z, u) \mapsto \theta(y, z, u)-l(y, z, u)$ est nul. Sinon ce n'est pas la restriction d'un germe de fonction de classe $C^{Q}$.

L'auteur remercie Jean-Philippe Rolin sans qui ce travail n'aurait pas existé.

\section{Bibliographie}

[BM] E. Bierstone et P. Milman, Semianalytic and subanalytic sets, Publ. IHES 67 (1988), 5-42.

[DM] L. van den Dries et C. Miller, Extending Tamm's theorem, Ann. Inst. Fourier (Grenoble) 44 (1994), 1367-1395.

[Ga] A. Gabrielov, Projections of semi-analytic sets, Functional Anal. Appl. 2 (1968), 282-291. 
[Ku] K. Kurdyka, Points réguliers d'un sous-analytique, Ann. Inst. Fourier (Grenoble) 38 (1988), 133-156.

[LR] J.-M. Lion et J.-P. Rolin, Théorème de préparation pour les fonctions logarithmico-exponentielles, ibid. 47 (1997), 859-884.

[Ło1] S. Łojasiewicz, Ensembles semi-analytiques, preprint I.H.E.S., 1965.

[Ło2] -, Triangulation of semi-analytic sets, Ann. Scuola Norm. Sup. Pisa (3) 18 (1964), 449-474.

[ETZ] S. Łojasiewicz, J.-C. Tougeron et M.-A. Zurro, Eclatements des coefficients des séries entières et deux théorèmes de Gabrielov, Manuscripta Math. 92 (1997), 325-337.

[Par] A. Parusiński, Lipschitz stratification of subanalytic sets, Ann. Ecole Norm. Sup. 27 (1994), 661-696.

[Paw] W. Pawłucki, Le théorème de Puiseux pour une application sous-analytique, Bull. Polish Acad. Sci. Math. 32 (1984), 555-559.

[Ta] M. Tamm, Subanalytic sets in the calculus of variation, Acta Math. 146 (1981), 167-199.

Laboratoire de Topologie

CNRS-UMR 5584

Université de Bourgogne

BP 400

21011 Dijon Cedex, France

E-mail: lion@u-bourgogne.fr 